\title{
Molecular Alterations in Patients with Pulmonary Adenocarcinoma Presenting with Malignant Pleural Effusion at the First Diagnosis
}

\author{
Erika F. Rodriguez ${ }^{\mathrm{a}} \quad$ Maryam Shabihkhani $^{\mathrm{a}} \quad$ Jamal Carter $^{\mathrm{b}}$ Zahra Maleki $^{\mathrm{a}}$

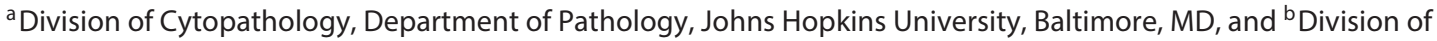 \\ Transfusion Medicine, Department of Pathology, National Institutes of Health, Bethesda, MD, USA
}

\section{Keywords}

Malignant pleural effusion · Molecular changes · Metastatic pulmonary adenocarcinoma

\begin{abstract}
Objectives: The aim of this study was to report cytologic and molecular features of pulmonary adenocarcinoma patients presenting with a malignant pleural effusion at the first diagnosis. Study Design: Patients who had a cytopathologic diagnosis conclusive for lung adenocarcinoma for the first time on their pleural fluid specimen, and molecular testing done, were studied. The control group consisted of patients with a malignant pleural effusion that developed during disease progression. Results: We identified 18 patients ( 9 males and 9 females). Micropapillary and/or solid adenocarcinoma type features predominated among cytologic specimens ( $n=15)$, while acinar patterns predominated in controls. Survival was not significantly different from that of the control group (mean 13.8 vs. 13.9 months, respectively; $p=0.61)$. Ten $(55 \%)$ cases had mutations in EGFR ( $n=6 ; 60 \%), \operatorname{KRAS}(n=3 ; 30 \%)$, or $A L K$ translocation ( $n=1 ; 10 \%)$. No mutations were identified in BRAF, AKT, ERBB2, NRAS, or PIK3CA (tested in 7 patients). Patients positive for the tested mutations had a better overall survival than patients negative for the mutations (mean survival 16.2 vs. 6.05 months, respectively; $p=0.006$, log-rank
\end{abstract}

(C) 2017 S. Karger AG, Basel test). Ten (84\%) control patients were positive for mutations in EGFR $(n=5 ; 42 \%), K R A S(n=4 ; 34 \%)$, or ALK translocation ( $n=1 ; 8.4 \%)$. Conclusion: In our series, a micropapillary-like and solid-like morphology, common in cytologic specimens, and alterations in EGFR were the most frequent identifiable molecular changes.

(c) 2017 S. Karger AG, Basel

\section{Introduction}

Pulmonary carcinoma is a heterogeneous group of neoplasms. Traditionally, it has been divided in 2 major groups: non-small cell lung carcinoma and small cell lung carcinoma [1]. Adenocarcinoma is the most common non-small cell lung carcinoma [1].

A better understanding of the mutational profile of this group of tumors has resulted in availability of many targeted therapies. Therefore, molecular studies are routinely performed to guide treatment, especially in advanced tumors [1, 2]. Nevertheless, despite advances in the field of thoracic oncology, lung cancer is still one of the major causes of cancer-related mortality worldwide [3], with the tumor stage being one of the strongest predictors of the prognosis [4].

Correspondence to: Dr. Erika F. Rodriguez 
Malignant pleural effusion (MPE) is defined by the presence of neoplastic cells on pleural fluid. MPE can occur as a complication of any malignancy. However, lung cancer is the most common cause of MPE [5-7]. The incidence of MPE in patients with lung cancer at any point during the course of the disease is estimated to be $7-23 \%$ [5-7], with pulmonary adenocarcinoma being the most common subtype [5-7]. However, the true incidence of patients presenting with MPE at the time of diagnosis of pulmonary adenocarcinoma is unknown. In a recent study of 556 patients with lung cancer, it was found that $26 \%$ of the patients had a pleural effusion at the time of diagnosis on imaging studies. However, in half of them the pleural effusion was too small to be aspirated [8]. Patients with MPE are classified as having stage IV disease in the tumor-node-metastasis staging system for lung cancer, with a poor survival rate [4].

The mechanism of pleural effusions in a setting of pulmonary malignancy, and its impact on survival is not well understood. Interestingly, not all malignancies with dissemination to the pleura cause MPE $[9,10]$. It has been suggested that these tumors may have a different molecular signature [11], and that the EGFR mutation is more common in pleural effusions than in primary tumors [12]. Nevertheless, the $K-R A S$ mutation has also been associated with pleural invasion [13].

Cytologic examination has become an important diagnostic tool not only to diagnose pulmonary cancer but also to help with tumor staging and collection of material for molecular studies. Several studies have been published on the utility of cytology specimens and molecular markers, especially in lung cancer [14-19]. However adequate cellularity remains a challenge, particularly in patients presenting with a first-time diagnosis of pulmonary adenocarcinoma as MPE. In these patients, the pleural effusion is the only material available for diagnosis and molecular testing. In the current study we focused on cytologic findings in a selected group of patients presenting with MPE of a presumptive lung primary with the aim of identifying typical morphologic features and molecular changes.

\section{Materials and Methods}

\section{Patient Selection}

A search on our electronic pathology database system was performed for patients with a cytopathologic diagnosis conclusive for metastatic pulmonary adenocarcinoma on their pleural fluid specimen between January 2010 and January 2015. Cases with MPE presenting at the time of the first diagnosis of pulmonary adeno- carcinoma and with molecular studies performed were included in this study. The control group consisted of patients with MPE that developed during the course of a previous diagnosis of pulmonary adenocarcinoma. Age, gender, ethnicity smoking history, molecular profile, survival, and history of thyrosine kinase inhibitor treatment were recorded. This study was conducted with the approval of the Institutional Review Board of Johns Hopkins Hospital, following all ethics guidelines and recommendations.

\section{Pleural Effusion Preparations}

All specimens were received fresh, without fixation, and they were prepared according to standard clinical protocols. The effusion preparation method is described in further detail in the paper by Rooper et al. [20]. In summary, depending on the volume, effusions were entirely submitted for centrifugation or a representative $100-\mathrm{mL}$ sample was processed. The specimens were divided into 2 tubes and centrifuged. One of the tubes was used to prepare the slides using the cytospin method (Thermo Fisher Scientific, Waltham, MA, USA) and stained with the Papanicolaou stain. A cell pellet was obtained from the other tube and the material was fixed in formalin, processed as a cell block, and stained with hematoxylin and eosin. Immunohistochemical staining was performed at the time of diagnosis on the cell block according to clinical protocols validated in our immunohistochemical laboratory.

\section{Molecular Testing}

Molecular testing was performed on formalin-fixed paraffinembedded blocks using various methods. Before February 2013, Sanger sequencing was used for detection of EGFR mutations within exons 18-21 and pyrosequencing was used to detect mutations at codons 12 and 13 of the KRAS gene and codon 600 of the $B R A F$ genes as previously described [21-23]. Reflex fluorescence in situ hybridization testing was performed to detect $A L K$ translocations if no mutations were identified in KRAS or EGFR.

After February 2013, next-generation sequencing was used for molecular testing. Next-generation sequencing was conducted using an AmpliSeq Cancer Hotspot Panel (version 2; Life Technologies, Carlsbad, CA, USA) for targeted multigene amplification as described in previous publications [22]. All protocols followed manufacturer's instructions and were DNA based. The DNA input for targeted multigene PCR was up to $30 \mathrm{ng}$ per sample. From January 2013 to April 2013 pulmonary adenocarcinomas were tested for BRAF, EGFR, and KRAS genes and reflex ALK FISH if no mutations were found on the genes previously cited. After April 2013, the panel included AKT, BRAF, EGFR, ERBB2, KRAS, NRAS, PIK $3 C A$, and $A L K$ (lung cancer panel).

\section{Morphologic Features}

All of the slides available were reviewed by 2 of the authors (E.F.R. and Z.M.). Neoplastic cells were analyzed for nuclear size, cytoplasm characteristics, cellular pleomorphism, nuclear contours, and the presence of nuclear membrane irregularity, the presence of nucleoli. Cases were considered to have a large nuclear size if the majority of the cells had nuclei larger than 5 times the size of a lymphocyte. Pleomorphism was considered to be present if the majority of the cells had marked variations in size and shape. Abnormal nuclear contours and the presence of nuclear irregularity were also considered present if they were marked and present in the majority of the cells. The cytoplasm was described as vacuolat- 


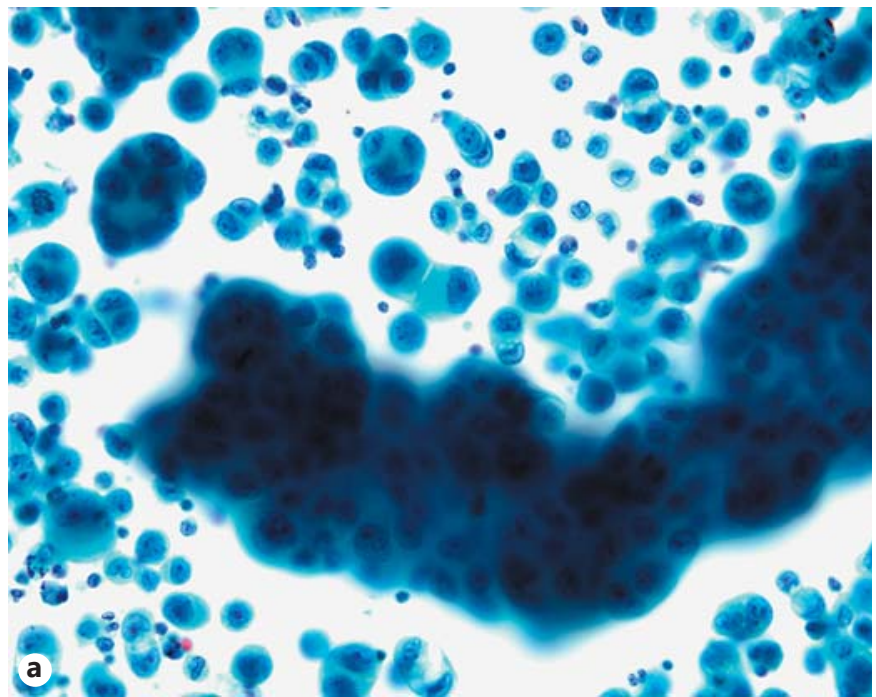

Fig. 1. a Cytologic features of metastatic pulmonary adenocarcinoma in pleural effusions. Clusters of cells showing a solid (3-dimensional clusters of cells with dense cytoplasm pleomorphic nuclei) and micropapillary-like architecture (tight small clusters of cells with predominantly eccentric nuclei). $\times 60$. Pap, cytospin. b Clusters of cells showing solid (i.e., nests of cells with well-defined cytoplasmic membranes; some of the cells have a dense cytoplasm and others show a clear cell change) with a micropapillary-like architecture. $\times 60$. Hematoxylin and eosin, cell block. c Immunohistochemical stain showing 3-dimensional clusters of cells (solid-like architecture) positive for TTF-1. $\times 40$.

ed, dense, granular, or other. The lymphocytic infiltrate was described as marked or mild/moderate.

An attempt to subtype the specimens using the most current histologic classification of pulmonary adenocarcinoma published by the International Association for the Study of Lung Cancer/ American Thoracic Society/European Respiratory Society/International Multidisciplinary Classification of Lung Adenocarcinoma (IASLC/ATS/ERS) and endorsed by the 2015 World Health Organization classification of lung cancer [1] was followed. Specifically, the various recognized patterns (acinar, lepidic, papillary, micropapillary, and solid) and cytologic criteria were recorded as outlined in our previous publications $[24,25]$. Briefly, the cytologic criteria for classification included the following: the acinar pattern consisted of glandular structures with central lumens; a solid pattern showed cohesive cell clusters in nests without identifiable lumens or a papillary configuration; a papillary pattern was defined as papillary structures with fibrovascular cores and occasional intranuclear inclusions; a micropapillary pattern was de-
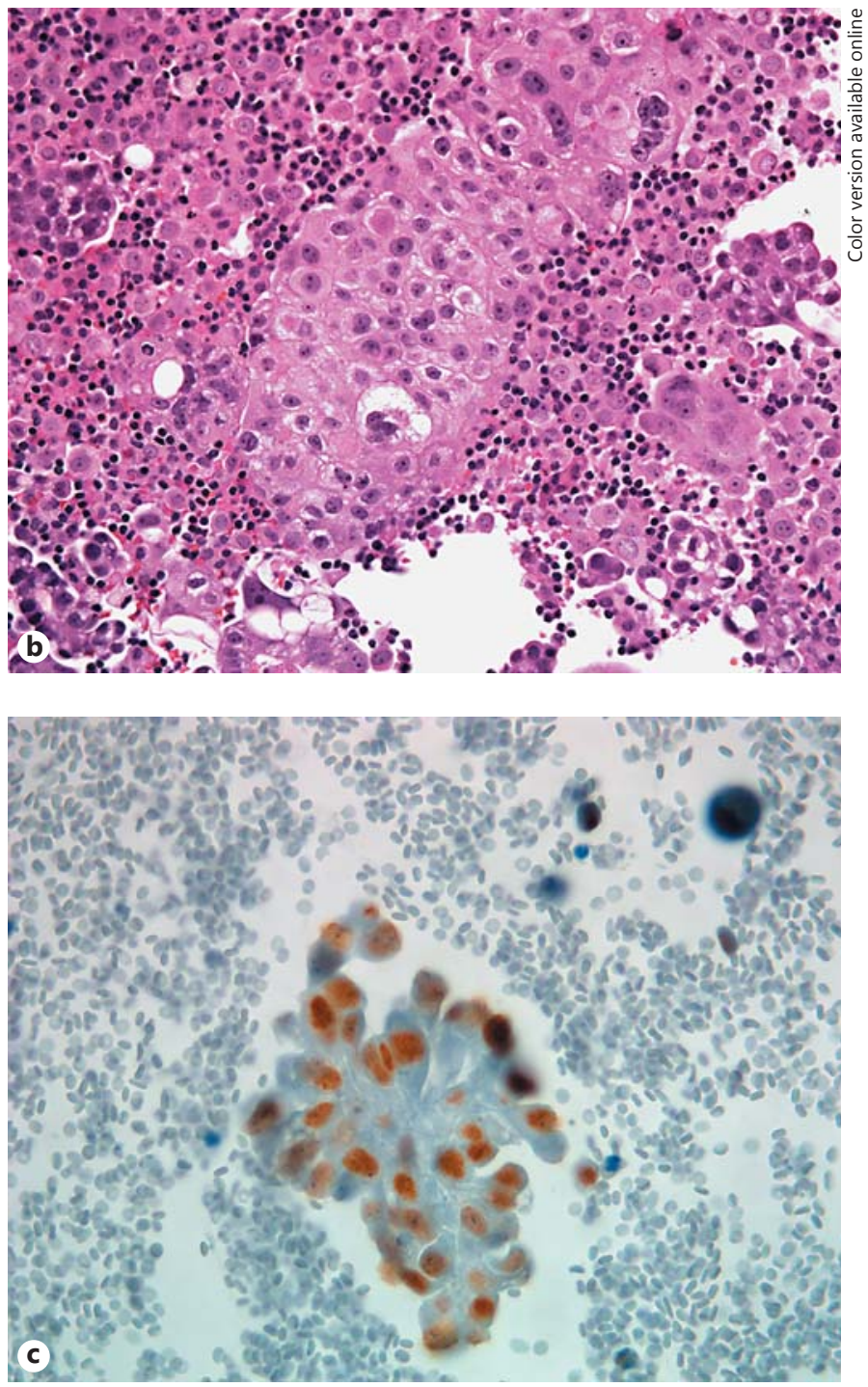

fined as micropapillary tuffs of small papillae without central fibrovascular cores and eccentric placed nuclei; mucinous adenocarcinomas had columnar cells with eccentric nuclei and intracytoplasmic or extracellular mucin; and a lepidic pattern was defined as strips of orderly arranged cells with uniform nuclei and intranuclear inclusions. Only the predominant patterns seen at a $\times 20$ magnification were recorded.

\section{Statistical Analysis}

A log-rank test and Kaplan-Meier curves were used to evaluate continuous variables and survival. Survival in the study and control groups was calculated from the date of cytologic diagnosis of a malignant effusion until the last follow-up. Fisher's exact test was used to compare categorical variables. All tests were 2 -sided, and $p<0.05$ considered statistically significant. Statistical tests were performed using JMP software version 10 (SAS Institute Inc.). 

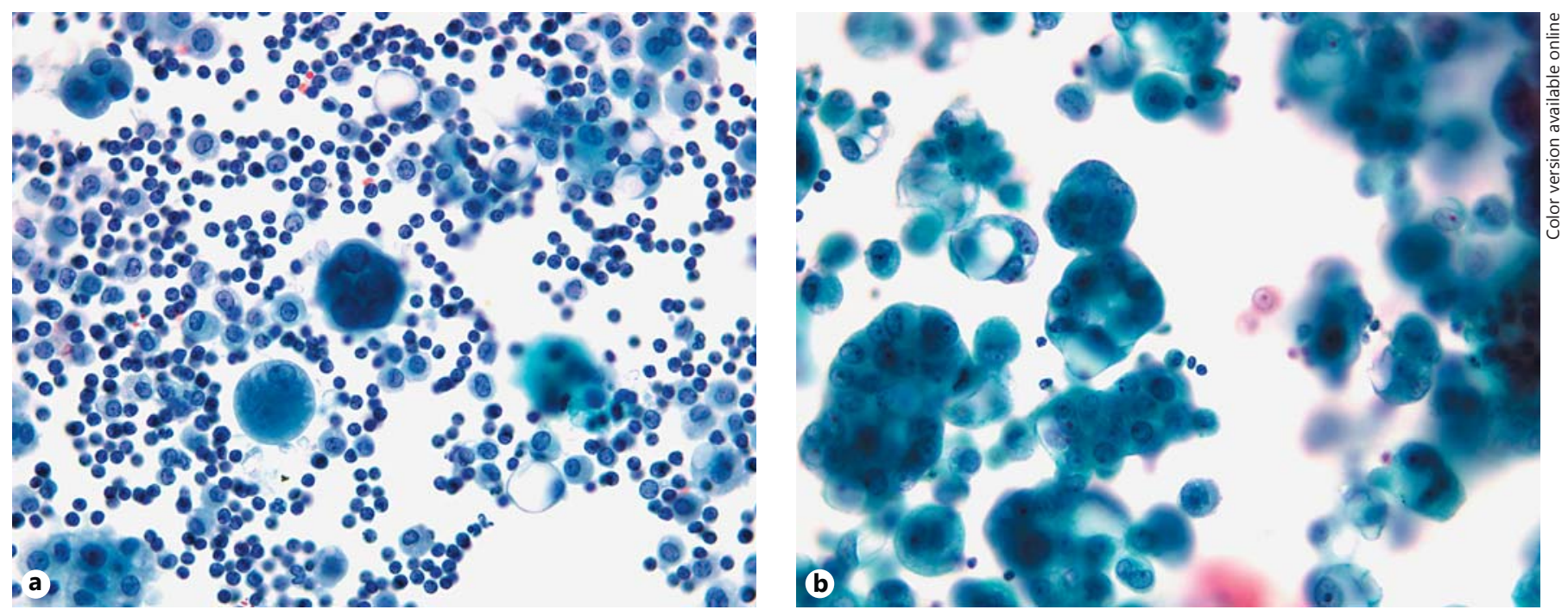

Fig. 2. a Cytologic features of metastatic pulmonary adenocarcinoma in pleural effusions. Clusters of cells showing a micropapillary-like (small tight "balls" of neoplastic cells with eccentric nuclei) architecture. $\times 60$. Pap, cytospin. $\mathbf{b}$ Clusters of cells showing a micropapillary-like architecture (small papillary structures lacking fibrovascular cores; the nuclei are eccentric). $\times 60$. Hematoxylin and eosin, cell block.

\section{Results}

\section{Patients}

We identified 18 cases ( 9 males and 9 females). The patients' mean age at the time of diagnosis was 69.6 years (range 55-81). Fifteen patients were Caucasian, 3 were African American, and none were Asian. Nine (50\%) of the patients were smokers, 7 (39\%) were nonsmokers, and $2(11 \%)$ had an unknown smoking history. Two (11\%) patients had a history of asbestos exposure and 3 (17\%) had previous malignancies in other organs. All of the patients had molecular tests performed on pleural effusions $(n=16$; $89 \%)$ or concurrent pleural biopsies $(n=2 ; 11 \%)$. For the control group, we identified 12 patients from the same database and time frame (6 males and 6 females), with a mean age at the time of the first MPE of 60.9 years (range 49-75). Ten patients were Caucasian, 1 was African American, and 1 was Asian. Two of the patients were smokers, 2 were former smokers, 9 were nonsmokers, and 1 had an unknown smoking history. Two patients had a history of other malignancies. All the patients had molecular tests performed on pleural effusions. Treatment was highly heterogeneous, including various chemotherapeutic regimens. In the study group with EGFR ( $n=6)$ mutations, 5 patients were treated with tyrosine kinase inhibitors and 1 was lost to follow-up. In the control group, 3 (of 5) patients with EGFR mutations were treated with tyrosine kinase inhibitors.

\section{Cytologic and Morphologic Characteristics}

Slides on 17 of the 18 cases were available for review. Pleomorphism was considered marked in 6 cases (35\%). Eight cases were considered to have a large nuclear size (47\%). Thirteen cases had nuclear contour irregularities (76\%) and 5 had marked nuclear membrane irregularities (29\%). The cytoplasm was considered mixed in 9 (53\%) cases (dense, vacuolated, and granular), dense in $6(35 \%)$ cases, and vacuolated in $2(12 \%)$ cases. Lymphocytic infiltrate was marked in $15(88 \%)$ cases. Most of the cases showed mixed micropapillary and solid morphological features $(n=9 ; 53 \%)$ (Fig. 1,2$)$. In 3 cases only micropapillary $(n=3 ; 18 \%)$ or a solid pattern $(n=3 ; 18 \%)$ was present. In $2(12 \%)$ cases cytologic subclassification was not possible due to a predominance of single cells. Although an acinar-like architecture was also noted in many cases, it was not a predominant feature.

Slides on 9 (of 12) cases were available for review in the control group. Pleomorphism was considered marked in $8(89 \%)$ cases. All of the cases had large nuclei, marked nuclear contour irregularities, and nuclear membrane irregularities $(n=9 ; 100 \%)$. A lymphocytic infiltrate was seen in 5 (56\%) cases. An acinar-like architecture was the 

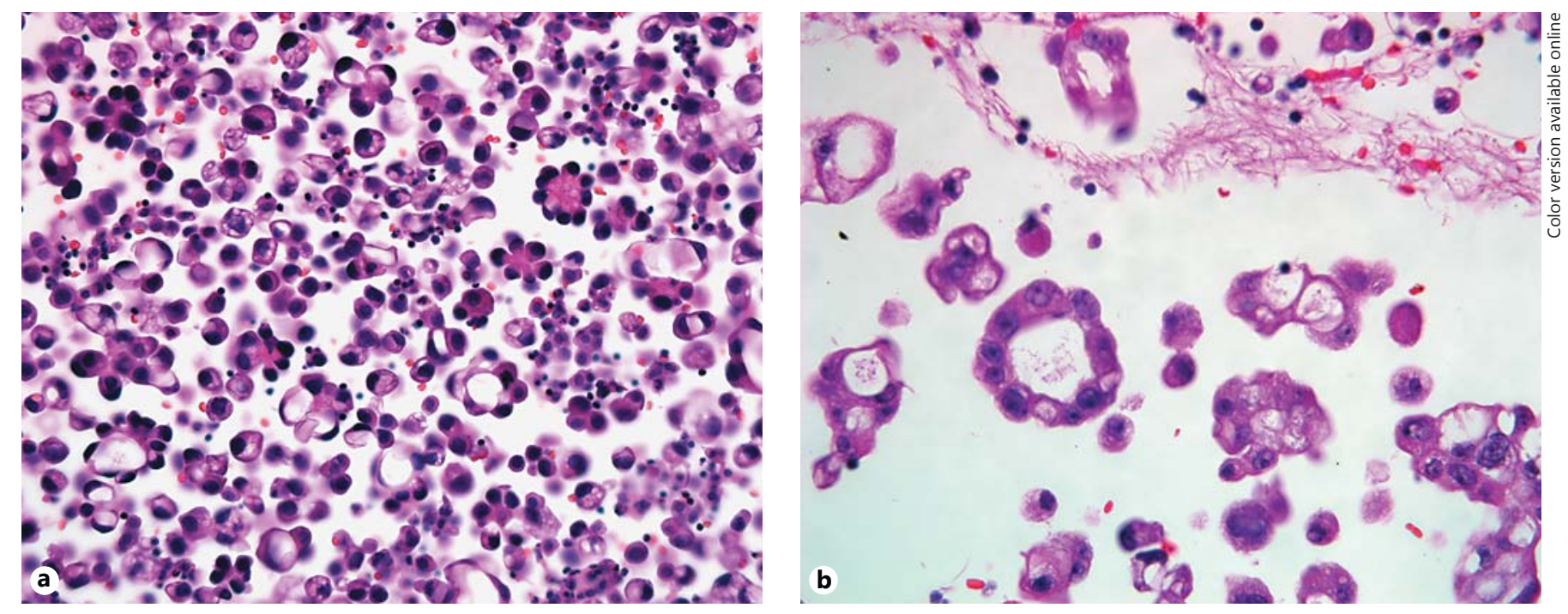

Fig. 3. a Cytologic features of metastatic pulmonary adenocarcinoma in pleural effusions following disease progression (control group). Cluster of neoplastic cells showing a glandular configuration and central lumen formation. $\times 60$. Pap, cytospin. $\mathbf{b}$ The same morphologic features are seen on the cell block of the corresponding case. $\times 60$. Hematoxylin and eosin, cell block.

predominant morphologic pattern in all of the cases in the control group $(n=9)$ (Fig. 3), which was statistically different when compared to the study group $(p>0.001$, Fisher's exact test). In association with the acinar-like morphology, 3 (34\%) cases showed a minor papillary-like component, 2 (34\%) showed a minor solid-like component, and 3 (34\%) showed a minor micropapillary component.

\section{Comparison with Concurrent Biopsies}

Only 2 patients in the study group had tissue obtained (pleural biopsy) in addition to the cytologic specimens, since all other 16 patients had enough material for molecular testing and were classified at the first diagnosis of lung cancer as having stage IV disease obviating the need for additional diagnostic procedures. One of these pleural biopsies demonstrated a predominance of solid-like and micropapillary-like architectural patterns. An acinar pattern was a minor component. In the corresponding cytology specimen, the micropapillary-like pattern was the only pattern identified, although the specimen was scant (Fig. 4). The pleural biopsy on the second patient was scant, showing an acinar/cribiform pattern only. The concurrent cytology specimen showed predominant solid-like and micropapillary-like patterns. Some acinarlike architecture was also seen.

\section{Immunohistochemical Studies}

All of the patients in the study group $(n=18)$ had TTF1 performed on the pleural effusion or a concurrent pleural biopsy. TTF-1 was positive in $15(83 \%)$ cases. Napsin A was performed in 12 cases and it was positive in $8(66 \%)$ cases. Three cases that were TTF-1 negative were negative for Napsin A as well. Cases with negative TTF-1 and Napsin A were considered pulmonary primary based on exclusion of other primaries and clinical diagnosis. In the control group TTF-1 was performed in 3 cases. They were all positive for TTF-1. Napsin A was not performed in any case.

\section{Molecular Studies}

In total, 18 cases were tested for lung adenocarcinoma-associated genes (e.g., EGFR, KRAS, and ALK) using the algorithm described in Materials and Methods. Mutations in one of these genes were identified in $10(55 \%)$ of the cases, including mutations in EGFR $(n=6 ; 33 \%)$, KRAS $(n=3 ; 16 \%)$, or ALK translocation $(n=1 ; 5.5 \%)$. Additionally, 7 of the 18 patients were tested for $B R A F$, $A K T, E R B B 2$, NRAS, and PIK3CA. No mutations were identified in these genes in the 7 patients tested for them.

In contrast, in the control group all 12 patients were tested for EGFR, KRAS, and ALK. Ten (84\%) patients were positive for mutations in EGFR $(n=5 ; 42 \%), K R A S$ ( $n=4 ; 34 \%)$, or $A L K$ translocation $(n=1 ; 8.4 \%)$. Four 


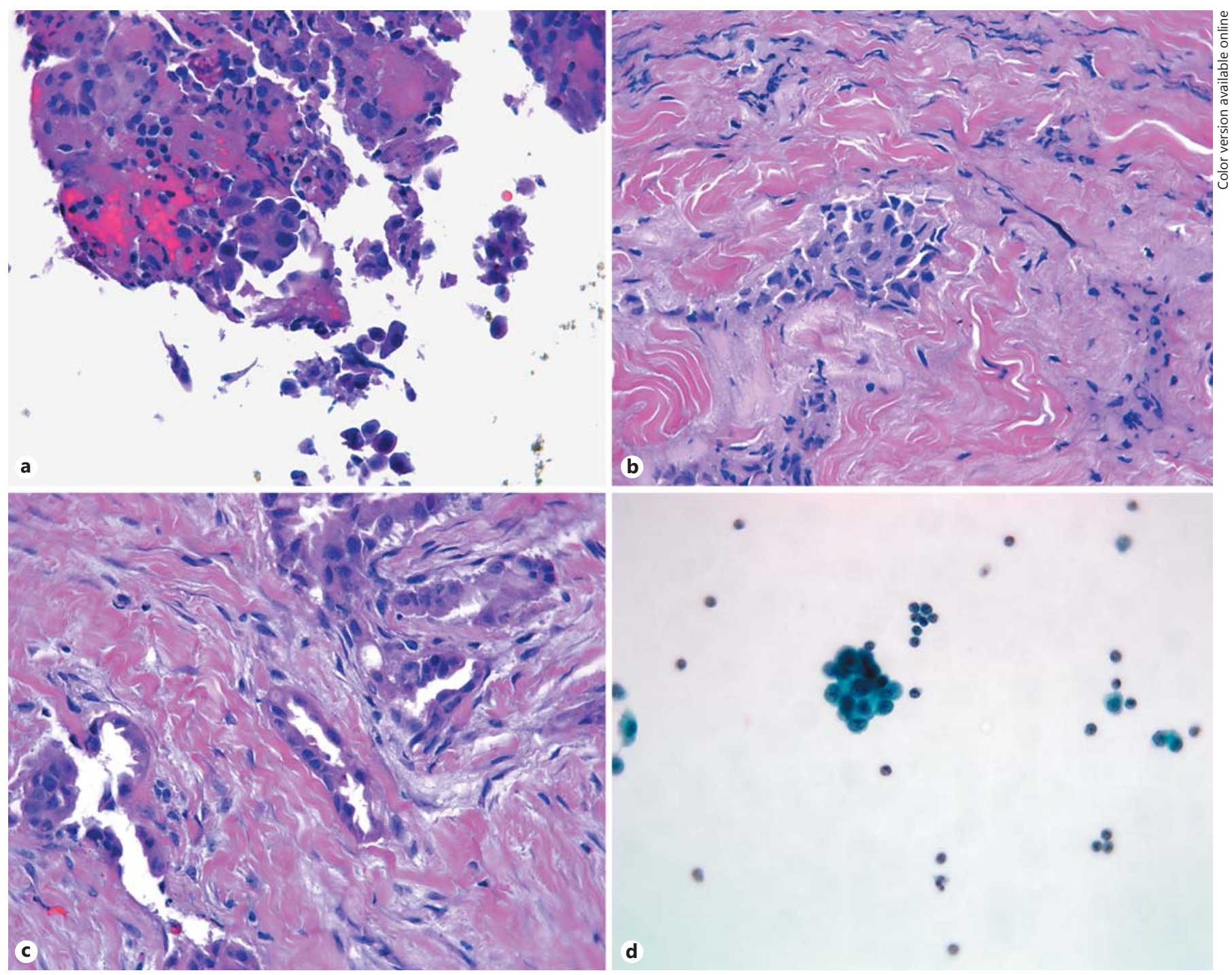

Fig. 4. a Morphologic and cytologic features of metastatic pulmonary adenocarcinoma in pleural biopsy and concurrent pleural effusion. Small papillary clusters of neoplastic cells lacking a fibrovascular core. Pleural biopsy. Hematoxylin and eosin. $\times 60$. b Nest of neoplastic cells growing in a "paving stone" fashion. Pleural bi-

opsy. Hematoxylin and eosin. $\times 60$. c Irregular glandular structures lined by neoplastic cells. Pleural biopsy. Hematoxylin and eosin. $\times 60$. d Corresponding effusion showing a micropapillary-like architecture (tight small clusters of cells with predominantly eccentric nuclei). $\times 60$. Pap, cytospin.

patients were also tested for BRAF, AKT, ERBB2, NRAS, and PIK3CA. One patient was positive for EGFR and $P I K 3 C A$. The remainder was negative for the tested genes.

\section{Treatment}

Of the 6 patients on the study group with the EGFR mutation, 5 were treated with tyrosine kinase inhibitors. One of the patients was lost to follow-up. In the control group, of the 5 patients with the EGFR mutation, 3 were treated with a tyrosine kinase inhibitor.

Molecular Alterations in MPE at the First Diagnosis

Acta Cytologica 2017;61:214-222

Survival Rates
Of the 18 patients in the study group, 2 were alive at the time of the last follow-up. The survival rate was calculated using either the date of death or the date of the last follow-up. The mean survival was 13.8 months, which was not significantly different from the value of the control group developing pleural effusions during the course of the disease (mean 13.9 months; $p=0.61$, log-rank test). In the study group, patients with known mutations had a better overall survival than patients without known mu- 
tations (mean survival 16.2 vs. 6.05 months, respectively; $p=0.006$, log-rank test). Patients with the EGFR mutation in particular had a better survival rate than patients without a mutation (mean survival 18.9 vs. 9.7 months, respectively; $p=0.03$, log-rank test). There was no association between morphologic features, specifically nuclear size and survival ( $p=0.85$, log-rank test) or nuclear size and tested mutations.

\section{Discussion}

Pulmonary adenocarcinoma is the most common cause of MPE [5-7]. It is an ominous sign and it is associated with a poor prognosis [5-7]. In patients with a pulmonary malignancy, the reported incidence of pleural effusion varies from 7 to $23 \%$ [5]. Patients with MPE are considered to have stage IV disease according to the 7 th edition of the tumor-node-metastasis staging system, with a 5 -years survival rate of $2 \%$ and a median survival of 8 months $[4,26]$.

In lung adenocarcinoma patients, a malignant effusion is an important specimen for molecular testing. If only tissue were used for molecular diagnosis, less than $50 \%$ of the patients would have enough material for successful molecular testing [27-29]. Molecular studies on MPE have a high concordance rate with tumor specimens. Thoracocentesis has some advantages over biopsy, for example it is an easier procedure and patients are more likely to accept recommendations of repeat sampling if needed [29].

MPE can be present at the initial diagnosis of pulmonary adenocarcinoma (patients presenting with MPE of unknown origin) or patients can develop MPE following disease progression. The true incidence of MPE at the initial diagnosis of lung cancer is not known. However, patients with MPE at the time of diagnosis have been reported to have a shorter survival than patients who develop MPE during the course of the disease (14.3 vs. 18.1 months, respectively) [29].

Although traditionally MPE has been explained by occlusion of parietal pleura and obstruction of mediastinal nodes, other features associated with tumor carcinogenesis may play a role. The EGFR mutation is the most common known mutation in MPE associated with lung cancer $[12,29,30]$. MPE is more common in patients in whom the primary tumor harbors the EGFR mutation. Also EGFR mutations are more common in pleural metastases and fluid compared to paired primary tumors [31]. Conversely, the frequency of the KRAS mutation is lower in MPE than in primary non-small cell lung carcinoma [32]. ALK fusions are the second most common mutation in patients with MPE [33].

Morphologic subtyping of adenocarcinomas using the current World Health Organization nomenclature proposed for surgical resection specimens is limited in routine cytologic specimens due to numerous challenges [24, 25]. Interestingly, all of the cases of patients presenting with MPE at the first diagnosis of pulmonary adenocarcinoma in our study had identifiable micropapillary and or a solid-like appearance, patterns that are associated with a worse prognosis in surgical specimens. In the control group an acinar-like morphology predominated. However, 3 of the cases showed an associated micropapillary architecture, and 2 others had solid-like components in association with the acinar-like pattern. Although the nomenclature as initially proposed was not directed to cytology or small biopsies [34], our group and others have studied its applicability to cytologic specimens, which has its challenges [35]. With this caveat, it was still interesting that a micropapillary-like architecture was the most frequent architectural pattern on the effusions of patients presenting with MPE, and it was present in 1 of the 2 biopsies available for comparison in this selected group of patients.

For practical purposes, recognition of these patterns may be useful for optimization of the triage of scant specimens, since in our study only $83 \%$ of the cases were positive for TTF-1 and 66\% were positive for Napsin A, and molecular studies are necessary at the present to guide targeted therapies. It is also important to note that in our study only 2 patients had concurrent tissue obtained, while the remainder had only pleural effusion material and no other concurrent or previous surgical pathology material. This highlights the rationale of identifying cytologic correlates of histologic patterns that are associated with different tumor subgroups, which may facilitate saving of material for further testing.

In our study, $55 \%(n=10)$ of the cases had identifiable lung adenocarcinoma-associated mutations, with EGFR being the most common (33\%), which is similar to frequencies reported in the literature (i.e., $42-50 \%$ ) [17]. In our study the rate of EGFR mutation in the study group was lower than the rates reported by $\mathrm{Wu}$ et al. [29] (i.e., $68.2 \%)$ and other authors $[32,36]$. In our study, patients with tumors containing EGFR mutations had a better survival as a group (18.5 months), which is also in agreement with the literature.

Regarding prognostic factors, patients with MPE at the initial diagnosis of lung adenocarcinoma have been re- 
ported in general to have a worse prognosis than the reported survival rate of patients developing MPE following disease progression (13.8 vs. 21.4 months in 1 study) [29], although we did not find a significant difference in our study, with the caveat that our study has a relative low number of cases. Also it has been reported that EGFR mutations in patients presenting with MPE are more frequent than in patients with MPE following disease progression [29].

In our study, there was no correlation between the morphologic features and survival or mutations, in contrast to lung cancer diagnosed on routine lung FNA and surgical specimens. This may be secondary in part to the uniform adverse clinical course of patients presenting with MPE at the first diagnosis. However, future studies should be useful to identify the clinicopathologic signifi- cance of this unique lung adenocarcinoma subgroup. In summary, we report cytologic and molecular findings in the subset of lung adenocarcinoma patients that present with MPE at the first diagnosis. Cytologic evaluation is critical in these patients given that they may represent the only specimens available for diagnosis and triage for immunohistochemical and molecular characterization. Cytologic features reminiscent of the prognostically adverse micropapillary and solid histologic patterns are almost uniformly present in these specimens, and this is a finding that deserves further study.

\section{Disclosure Statement}

The authors have no financial conflicts of interest to disclose.

\section{References}

1 Travis WD, Brambilla EBP, Marx A, Nicholson A: WHO Classification of Tumours of the Lung, Pleura, Thymus and Heart, ed 4. Lyon, WHO, 2015.

2 Lindeman NI, Cagle PT, Beasley MB, Chitale DA, Dacic S, Giaccone G, Jenkins RB, Kwiatkowski DJ, Saldivar JS, Squire J, Thunnissen E, Ladanyi M: Molecular testing guideline for selection of lung cancer patients for EGFR and ALK tyrosine kinase inhibitors: guideline from the College of American Pathologists, International Association for the Study of Lung Cancer, and Association for Molecular Pathology. Arch Pathol Lab Med 2013;137: 828-860.

3 Siegel RL, Miller KD, Jemal A: Cancer statistics, 2015. CA Cancer J Clin 2015;65:5-29.

4 Eberhardt WE, Mitchell A, Crowley J, Kondo H, Kim YT, Turrisi A 3rd, Goldstraw P, RamiPorta R; International Association for Study of Lung Cancer Staging and Prognostic Factors Committee, Advisory Board Members, and Participating Institution: The IASLC Lung Cancer Staging Project: Proposals for the revision of the $\mathrm{M}$ descriptors in the forthcoming eighth edition of the TNM classification of lung cancer. J Thorac Oncol 2015;10: 1515-1522.

5 Johnston WW: The malignant pleural effusion: a review of cytopathologic diagnoses of 584 specimens from 472 consecutive patients. Cancer 1985;56:905-909.

6 Sahn SA: Malignancy metastatic to the pleura. Clin Chest Med 1998;19:351-361.

7 Porcel JM, Esquerda A, Vives M, Bielsa S: Etiology of pleural effusions: analysis of more than 3,000 consecutive thoracenteses. Archivos Bronconeumol 2014;50:161-165.
8 Porcel JM, Gasol A, Bielsa S, Civit C, Light RW, Salud A: Clinical features and survival of lung cancer patients with pleural effusions. Respirology 2015;20:654-659.

9 Egan AM, McPhillips D, Sarkar S, Breen DP: Malignant pleural effusion. QJM 2014;107: 179-184.

10 Stathopoulos GT, Kalomenidis I: Malignant pleural effusion: tumor-host interactions unleashed. Am J Respir Crit Care Med 2012;186: 487-492.

11 Froudarakis ME: Pleural effusion in lung cancer: more questions than answers. Respiration 2012;83:367-376.

12 Zou J, Bella AE, Chen Z, Han X, Su C, Lei Y, Luo $\mathrm{H}$ : Frequency of EGFR mutations in lung adenocarcinoma with malignant pleural effusion: implication of cancer biological behaviour regulated by EGFR mutation. J Int Med Res 2014;42:1110-1117.

13 Raparia K, Villa C, Raj R, Cagle PT: Peripheral lung adenocarcinomas with KRAS mutations are more likely to invade visceral pleura. Arch Pathol Lab Med 2015;139:189-193.

14 Wang W, Tang Y, Li J, Jiang L, Jiang Y, Su X: Detection of ALK rearrangements in malignant pleural effusion cell blocks from patients with advanced non-small cell lung cancer: a comparison of Ventana immunohistochemistry and fluorescence in situ hybridization. Cancer Cytopathol 2015;123:117-122.

15 da Cunha Santos G, Saieg MA: Cell blocks for subtyping and molecular studies in non-small cell lung carcinoma. Cytopathology 2015;26: 331-333.
16 Rekhtman N, Brandt SM, Sigel CS, Friedlander MA, Riely GJ, Travis WD, Zakowski MF, Moreira AL: Suitability of thoracic cytology for new therapeutic paradigms in non-small cell lung carcinoma: high accuracy of tumor subtyping and feasibility of EGFR and KRAS molecular testing. J Thorac Oncol 2011;6: 451-458.

17 Akamatsu H, Koh Y, Kenmotsu H, Naito T, Serizawa M, Kimura M, Mori K, Imai H, Ono A, Shukuya T, Taira T, Murakami H, Ohde Y, Endo M, Nakajima T, Takahashi T, Yamamoto N: Multiplexed molecular profiling of lung cancer using pleural effusion. J Thorac Oncol 2014;9:1048-1052.

18 Fassina A, Gazziero A, Zardo D, Corradin M, Aldighieri E, Rossi GP: Detection of EGFR and KRAS mutations on trans-thoracic needle aspiration of lung nodules by high resolution melting analysis. J Clin Pathol 2009;62: 1096-1102.

19 Wei EX, Anga AA, Martin SS, Jackson JG, Nordberg ML, Herrera GA, Turbat-Herrera EA: EGFR expression as an ancillary tool for diagnosing lung cancer in cytology specimens. Mod Pathol 2007;20:905-913.

20 Rooper LM, Ali SZ, Olson MT: A minimum fluid volume of $75 \mathrm{~mL}$ is needed to ensure adequacy in a pleural effusion: a retrospective analysis of 2,540 cases. Cancer Cytopathol 2014; 122:657-665

21 Tsiatis AC, Norris-Kirby A, Rich RG, Hafez MJ, Gocke CD, Eshleman JR, Murphy KM: Comparison of Sanger sequencing, pyrosequencing, and melting curve analysis for the detection of KRAS mutations: diagnostic and clinical implications. J Mol Diagn 2010;12: 425-432. 
22 Lin MT, Mosier SL, Thiess M, Beierl KF, Debeljak M, Tseng LH, Chen G, Yegnasubramanian S, Ho H, Cope L, Wheelan SJ, Gocke CD, Eshleman JR: Clinical validation of KRAS, BRAF, and EGFR mutation detection using next-generation sequencing. Am J Clin Pathol 2014;141:856-866

23 Munfus-McCray D, Harada S, Adams C, Askin F, Clark D, Gabrielson E, Li QK: EGFR and KRAS mutations in metastatic lung adenocarcinomas. Hum Pathol 2011;42:14471453.

24 Rodriguez EF, Dacic S, Pantanowitz L, Khalbuss WE, Monaco SE: Cytopathology of pulmonary adenocarcinoma with a single histological pattern using the proposed International Association for the Study of Lung Cancer/American Thoracic Society/European Respiratory Society (IASLC/ATS/ERS) classification. Cancer Cytopathol 2015;123: 306-317.

25 Rodriguez EF, Monaco SE, Dacic S: Cytologic subtyping of lung adenocarcinoma by using the proposed International Association for the Study of Lung Cancer/American Thoracic Society/European Respiratory Society (IASLC/ATS/ERS) adenocarcinoma classification. Cancer Cytopathol 2013;121:629-637.

26 Goldstraw P, Crowley J, Chansky K, Giroux DJ, Groome PA, Rami-Porta R, Postmus PE, Rusch V, Sobin L; International Association for the Study of Lung Cancer International Staging Committee; Participating Institutions: The IASLC Lung Cancer Staging Project: proposals for the revision of the TNM stage groupings in the forthcoming (seventh) edition of the TNM Classification of malignant tumours. J Thorac Oncol 2007;2:706714.

27 Mok TS, Wu YL, Thongprasert S, Yang CH, Chu DT, Saijo N, Sunpaweravong P, Han B, Margono B, Ichinose Y, Nishiwaki Y, Ohe Y, Yang JJ, Chewaskulyong B, Jiang H, Duffield EL, Watkins CL, Armour AA, Fukuoka M: Gefitinib or carboplatin-paclitaxel in pulmonary adenocarcinoma. N Engl J Med 2009; 361:947-957.

28 Pao W, Chmielecki J: Rational, biologically based treatment of EGFR-mutant non-smallcell lung cancer. Nat Rev Cancer 2010;10: 760-774.

29 Wu SG, Yu CJ, Tsai MF, Liao WY, Yang CH, Jan IS, Yang PC, Shih JY: Survival of lung adenocarcinoma patients with malignant pleural effusion. Eur Respir J 2013;41:1409-1418.

30 Wu SG, Gow CH, Yu CJ, Chang YL, Yang CH, Hsu YC, Shih JY, Lee YC, Yang PC: Frequent epidermal growth factor receptor gene mutations in malignant pleural effusion of lung adenocarcinoma. Eur Respir J 2008;32:924-930.

31 Han HS, Eom DW, Kim JH, Kim KH, Shin HM, An JY, Lee KM, Choe KH, Lee KH, Kim ST, Koo JH, Lee HC, Lee OJ: EGFR mutation status in primary lung adenocarcinomas and corresponding metastatic lesions: discordance in pleural metastases. Clin Lung Cancer 2011;12:380-386.

32 Smits AJ, Kummer JA, Hinrichs JW, Herder GJ, Scheidel-Jacobse KC, Jiwa NM, Ruijter TE, Nooijen PT, Looijen-Salamon MG, Ligtenberg MJ, Thunnissen FB, Heideman DA, de Weger RA, Vink A: EGFR and KRAS mu- tations in lung carcinomas in the Dutch population: increased EGFR mutation frequency in malignant pleural effusion of lung adenocarcinoma. Cell Oncol (Dordr) 2012;35:189196.

33 Wu SG, Kuo YW, Chang YL, Shih JY, Chen $\mathrm{YH}$, Tsai MF, Yu CJ, Yang $\mathrm{CH}$, Yang PC: EML4-ALK translocation predicts better outcome in lung adenocarcinoma patients with wild-type EGFR. J Thorac Oncol 2012;7:98104.

34 Travis WD, Brambilla E, Noguchi M, Nicholson AG, Geisinger K, Yatabe Y, Ishikawa Y, Wistuba I, Flieder DB, Franklin W, Gazdar A, Hasleton PS, Henderson DW, Kerr KM, Petersen I, Roggli V, Thunnissen E, Tsao M: Diagnosis of lung cancer in small biopsies and cytology: implications of the 2011 International Association for the Study of Lung Cancer/American Thoracic Society/European Respiratory Society classification. Arch Pathol Lab Med 2013;137:668-684.

35 Sigel CS, Rudomina DE, Sima CS, Rekhtman $\mathrm{N}$, Travis WD, Geisinger KR, Moreira AL: Predicting pulmonary adenocarcinoma outcome based on a cytology grading system. Cancer Cytopathol 2012;120:35-43.

36 Akamatsu H, Kaira K, Murakami H, Serizawa M, Koh Y, Ono A, Shukuya T, Tsuya A, Nakamura Y, Kenmotsu H, Naito T, Takahashi T, Endo M, Harada H, Nakajima T, Yamamoto N: The impact of clinical outcomes according to EGFR mutation status in patients with locally advanced lung adenocarcinoma who received concurrent chemoradiotherapy. Am J Clin Oncol 2014;37:144-147. 